\title{
Deep Inelastic Scattering at the Amplitude Level
}

\author{
Stanley J. Brodsky \\ Stanford Linear Accelerator Center, Stanford University, Stanford, CA, 94309
}

\begin{abstract}
The deep inelastic lepton scattering and deeply virtual Compton scattering cross sections can be interpreted in terms of the fundamental wavefunctions defined by the light-front Fock expansion, thus allowing tests of QCD at the amplitude level. The AdS/CFT correspondence between gauge theory and string theory provides remarkable new insights into QCD, including a model for hadronic wavefunctions which display conformal scaling at short distances and color confinement at large distances.
\end{abstract}

Keywords: Quantum Chromodynamics, Deep Inelastic Scattering, Gauge/String Duality PACS: $12.38 . \mathrm{Aw}, 13.60 . \mathrm{Hb}, 11.25 . \mathrm{Tq}$

\section{WAVEFUNCTION REPRESENTATION OF DIS AND DVCS}

The primary goal of deep inelastic lepton scattering is to resolve the fundamental structure of the nucleon. In fact, by combining measurements of DIS with measurements of deeply virtual Compton scattering, elastic lepton-hadron scattering, and other hard exclusive channels, it is possible to obtain information on the fundamental form of quark and gluon bound-state wavefunctions. Thus, for the first time, we have the potential to test QCD at the amplitude level.

If one quantizes QCD at fixed light-front time $x^{+}=x^{0}+x^{3}$, the bound state hadronic solutions $\left|\Psi_{H}\right\rangle$ are eigenstates of the light-front Heisenberg equation $H_{L F}\left|\Psi_{H}\right\rangle=$ $M_{H}^{2}\left|\Psi_{H}\right\rangle$ [1]. The spectrum of QCD is given by the eigenvalues $M_{H}^{2}$. The projection of each hadronic eigensolution on the free Fock basis: $\left\langle n \mid \Psi_{H}\right\rangle \equiv \psi_{n / H}\left(x_{i}, \vec{k}_{\perp i}, \lambda_{i}\right)$ defines the LF Fock expansion in terms of the quark and transversely polarized gluon constituents in $A^{+}=0$ light-cone gauge. The light-front wavefunctions are frameindependent functions of the constituent light-cone fractions $x_{i}$, relative transverse momenta $\vec{k}_{\perp i}$, and spin projections $S_{i}^{z}=\lambda_{i}$. Observables in DIS and DVCS can be calculated directly from the hadron LFWFs. For example, the quark and gluon distributions measured in DIS are defined from the squares of the LFWFS summed over all Fock states $n$. Form factors, exclusive weak transition amplitudes [2] and the generalized parton distributions [3] measured in DVCS are overlaps of the initial and final LFWFS with $n=n^{\prime}$ and $n=n^{\prime}+2$. The resulting distributions obey DGLAP, BFKL, and ERBL evolution as a function of the maximal invariant mass, thus providing a physical factorization scheme [4]. It is important to note that at large $x$ where the struck quark is far-off shell, DGLAP evolution is quenched [5], so that the fall-off of the DIS cross sections in $Q^{2}$ satisfies inclusive-exclusive duality at fixed $W^{2}$. The gauge-invariant distribution amplitude $\phi_{H}\left(x_{i}, Q\right)$ defined from the integral over the transverse momenta $\vec{k}_{\perp i}^{2} \leq Q^{2}$ of the valence (smallest $n$ ) Fock state provides a fundamental measure of the hadron at the amplitude level $[6,7]$; they are the nonperturbative input to the factorized form of hard 
exclusive amplitudes and exclusive heavy hadron decays in PQCD. The front form provides a consistent definition of relative orbital angular momentum and $J^{z}$ conservation. Fundamental sum rules such as Ji's measure of orbital angular momentum [8], and the vanishing of the "anomalous gravitomagnetic moment" $B(0)$ [9] are immediate properties of the LF Fock wavefunctions [10]. One can perform Fourier transforms of the Fock state wavefunctions in impact space $b_{\perp}$ and in $x^{-}=x^{0}-x^{3}$ space to obtain the spatial form of Fock wavefunctions in coordinate space. See also: [11, 12] The DVCS amplitudes also enter the two-photon exchange contribution to elastic electron-proton scattering, which in turn produces a significant correction to the Rosenbluth method used for separating form factors $[13,14]$.

The E791 experiments at Fermilab [15] has shown how one can measure the valence LFWF directly from the diffractive di-jet dissociation of a high energy pion $\pi A \rightarrow q \bar{q} A^{\prime}$ into two jets, nearly balancing in transverse momentum, leaving the nucleus intact. The measured pion distribution in $x$ and $(1-x)$ is similar the form of the asymptotic distribution amplitude. The E791 experiment also find that the nuclear amplitude is additive in the number of nucleons when the quark jets are produced at high $k_{\perp}$, thus giving a dramatic confirmation of "color transparency", a fundamental manifestation of the gauge nature of QCD [16, 17] The LFWFs display other novel features, such as asymmetric sea-quark distributions $\bar{u}(x) \neq \bar{d}(x), \bar{s}(x) \neq s(x)$, and intrinsic heavyquark Fock states [18] of the proton $|u u d Q \bar{Q}\rangle$ in which the heavy constituents carry the largest moment fractions. One can use the OPE to show that the probability of such states scales as $1 / M_{O}^{2}$ in contrast to $1 / M_{\ell}^{4}$ fall-off of abelian theory [19]. The remarkable observations of the SELEX experiment of the double-charm baryon $\Xi_{c c d}$ in $p A \rightarrow \Xi_{c c d} X$ and $\Sigma^{-} A \rightarrow \Xi_{c c d} X$ at large $x_{F}$ [20] provides compelling evidence for double-charm intrinsic Fock states in the proton. The coherence of multi-particle correlations within the Fock states leads to higher-twist bosonic processes such as $e(q q) \rightarrow e^{\prime}(q q)^{\prime}$; although suppressed by inverse powers of $Q^{2}$, such subprocesses are important in the duality regime of fixed $W^{2}$, particularly in $\sigma_{L}$ [21]. In the case of nuclei, one must include nonnucleonic "hidden color" [22] degrees of freedom of the deuteron LFWF.

Contrary to parton model expectations, the rescattering of the quarks in the final state in DIS has important phenomenological consequences, such as leading-twist diffractive DIS [23] and the Sivers single-spin asymmetry [24]. The Sivers asymmetry depends on the same matrix elements which produce the anomalous magnetic moment of the target nucleon as well as the phase difference of the final-state interactions in different partial waves. The rescattering of the struck parton generates dominantly imaginary diffractive amplitudes, giving rise to an effective "hard pomeron" exchange and a rapidity gap between the target and diffractive system, while leaving the target intact. This Bjorkenscaling physics, which is associated with the Wilson line connecting the currents in the virtual Compton amplitude survives even in light-cone gauge. Thus there are contributions to the DIS structure functions which are not included in the light-front wave functions computed in isolation and cannot be interpreted as parton probabilities [23]. DDIS in turn leads to nuclear shadowing at leading twist as a result of the destructive interference of multi-step processes within the nucleus. In addition, multi-step processes involving Reggeon exchange leads to antishadowing. In fact, because Reggeon couplings are flavor specific, antishadowing is predicted to be non-universal, depending on the type of current and even the polarization of the probes in nuclear DIS [25]. Another 
particularly interesting consequence of QCD is the $Q^{2}$-independent " $J=0$ fixed-pole" contribution $M\left(\gamma^{*} p \rightarrow \gamma p\right) \sim s^{0} F(t)$ to the real part of the DVCS amplitude, reflecting the effective contact interaction of the transverse currents [26]. DVCS can also be studied in the timelike domain from $e^{+} e^{-} \rightarrow H^{+} H^{-} \gamma$; the lepton charge asymmetry and single-spin asymmetries allow measurements of the relative phase of timelike form factors and the $\gamma^{*} \rightarrow H^{+} H^{-} \gamma$ amplitude [27].

\section{ADS/CFT PREDICTIONS FOR HADRON PHYSICS}

The AdS/CFT correspondence [28], between strongly-coupled conformal gauge theory and weakly-coupled string theory in the 10-dimensional $A d S_{f} \times S^{5}$ space is now providing a remarkable new insight into hadron wavefunctions of QCD. Although QCD is not conformal, it is nearly conformal in the asymptotic freedom domain and it resembles a strongly-coupled conformal theory at relatively soft momenta if the QCD coupling has an infrared fixed point [29]. Deur et al. have also shown that the effective charge $\alpha_{g_{1}}^{s}\left(Q^{2}\right)$ defined from the radiative corrections to the Bjorken sum rule also approaches a constant at low momentum [30]. The near-constancy of the effective QCD coupling at small scales helps explain the empirical success of dimensional counting rules for the power law fall-off of form factors and fixed angle scaling. The string/gauge theory duality provides a framework for predicting QCD phenomena based on the conformal properties of the AdS/CFT correspondence. For example, the dimensional counting rules for the power-law fall-off of hard exclusive hadron-hadron scattering amplitudes at large momentum transfer can be derived [31, 32, 33] without the use of perturbation theory. Polchinski and Strassler [31] have also derived counting rules for deep inelastic structure functions at $x \rightarrow 1$ in agreement with perturbative QCD predictions [34]

Recently Teramond and I $[35,36,37]$ have shown how to compute the hadronic spectrum of light $q \bar{q}, q q q$ and $g g$ bound states in a holographic dual of QCD defined on $A d S_{5} \times S^{5}$. Specific hadrons are identified by the correspondence of string modes with the dimension of the interpolating operator of the hadron's valence Fock state, including orbital angular momentum excitations. Since only one parameter, the QCD scale $\Lambda_{Q C D}$, is introduced, the agreement with the pattern of physical states is remarkable. In particular, the ratio of $\Delta$ to nucleon trajectories is determined by the ratio of zeros of Bessel functions We have also shown how one can use the extended AdS/CFT space-time theory to obtain a model for the form of hadron LFWFs. The model wavefunctions display confinement at large inter-quark separation and conformal symmetry at short distances. In particular, the scaling and conformal properties of the LFWFs at high relative momenta agree with perturbative QCD [38]. These AdS/CFT model wavefunctions could be used as an initial ansatz for a variational treatment of the light-front QCD Hamiltonian.

\section{ACKNOWLEDGMENTS}

This work was supported by the Department of Energy, contract No. DE-AC0276 SF00515. 


\section{REFERENCES}

1. S. J. Brodsky, H. C. Pauli and S. S. Pinsky, Phys. Rept. 301, 299 (1998) [arXiv:hep-ph/9705477].

2. S. J. Brodsky and D. S. Hwang, Nucl. Phys. B 543, 239 (1999) [arXiv:hep-ph/9806358].

3. S. J. Brodsky, M. Diehl and D. S. Hwang, Nucl. Phys. B 596, 99 (2001) [arXiv:hep-ph/0009254].

4. G. P. Lepage and S. J. Brodsky, Phys. Rev. D 22, 2157 (1980).

5. S. J. Brodsky and G. P. Lepage, SLAC-PUB-2294

6. G. P. Lepage and S. J. Brodsky, Phys. Lett. B 87, 359 (1979).

7. A. V. Efremov and A. V. Radyushkin, Phys. Lett. B 94, 245 (1980).

8. X. D. Ji, Phys. Rev. Lett. 78, 610 (1997) [arXiv:hep-ph/9603249].

9. O. V. Teryaev, arXiv:hep-ph/9904376.

10. S. J. Brodsky, D. S. Hwang, B. Q. Ma and I. Schmidt, Nucl. Phys. B 593, 311 (2001) [arXiv:hepth/0003082].

11. X. Ji, Ann. Rev. Nucl. Part. Sci. 54, 413 (2004).

12. M. Burkardt, arXiv:hep-ph/0505189.

13. A. V. Afanasev, S. J. Brodsky, C. E. Carlson, Y. C. Chen and M. Vanderhaeghen, arXiv:hep$\mathrm{ph} / 0502013$.

14. Y. C. Chen, A. Afanasev, S. J. Brodsky, C. E. Carlson and M. Vanderhaeghen, Phys. Rev. Lett. 93, 122301 (2004) [arXiv:hep-ph/0403058].

15. D. Ashery [E791 Collaboration], arXiv:hep-ex/0205011.

16. G. Bertsch, S. J. Brodsky, A. S. Goldhaber and J. F. Gunion, Phys. Rev. Lett. 47, 297 (1981).

17. S. J. Brodsky and A. H. Mueller, Phys. Lett. B 206, 685 (1988).

18. S. J. Brodsky, P. Hoyer, C. Peterson and N. Sakai, Phys. Lett. B 93, 451 (1980).

19. M. Franz, . V. Polyakov and K. Goeke, Phys. Rev. D 62, 074024 (2000) [arXiv:hep-ph/0002240].

20. J. Engelfried [SELEX Collaboration], Nucl. Phys. A 752, 121 (2005).

21. S. J. Brodsky, E. L. Berger and G. P. Lepage, SLAC-PUB-3027

22. S. J. Brodsky, C. R. Ji and G. P. Lepage, Phys. Rev. Lett. 51, 83 (1983).

23. S. J. Brodsky, P. Hoyer, N. Marchal, S. Peigne and F. Sannino, Phys. Rev. D 65, 114025 (2002) [arXiv:hep-ph/0104291].

24. S. J. Brodsky, D. S. Hwang and I. Schmidt, Phys. Lett. B 530, 99 (2002) [arXiv:hep-ph/0201296].

25. S. J. Brodsky, I. Schmidt and J. J. Yang, Phys. Rev. D 70, 116003 (2004) [arXiv:hep-ph/0409279].

26. S. J. Brodsky, F. E. Close and J. F. Gunion, Phys. Rev. D 5, 1384 (1972).

27. A. V. Afanasev, S. J. Brodsky, C. E. Carlson, (in prepration).

28. J. M. Maldacena, Adv. Theor. Math. Phys. 2, 231 (1998) [Int. J. Theor. Phys. 38, 1113 (1999)] [arXiv:hep-th/9711200].

29. For a review and references see: S. J. Brodsky, arXiv:hep-ph/0408069.

30. A. Deur, et al. (This conference)

31. J. Polchinski and M. J. Strassler, Phys. Rev. Lett. 88, 031601 (2002) [arXiv:hep-th/0109174].

32. R. C. Brower and C. I. Tan, Nucl. Phys. B 662, 393 (2003) [arXiv:hep-th/0207144].

33. O. Andreev, Phys. Rev. D 67, 046001 (2003) [arXiv:hep-th/0209256].

34. S. J. Brodsky, M. Burkardt and I. Schmidt, Nucl. Phys. B 441, 197 (1995) [arXiv:hep-ph/9401328].

35. G. F. de Teramond and S. J. Brodsky, Phys. Rev. Lett. 94, 201601 (2005) [arXiv:hep-th/0501022].

36. G. F. de Teramond and S. J. Brodsky, arXiv:hep-th/0409074.

37. S. J. Brodsky and G. F. de Teramond, Phys. Lett. B 582, 211 (2004) [arXiv:hep-th/0310227].

38. X. d. Ji, J. P. Ma and F. Yuan, Phys. Rev. Lett. 90, 241601 (2003) [arXiv:hep-ph/0301141]. 